\title{
Incidence of Central Line-Associated Blood Steam Infection in Pediatric Intensive Care Unit (PICU) \\ Hanan M. Abdelmoneim ${ }^{1}$, Hanan M. Ibrahim ${ }^{2}$, Ahmed R. Ahmed ${ }^{2}$, Khaled A. Mohammed ${ }^{1}$ \\ 1 -Department of Pediatrics, Faculty of Medicine, Aswan University, 2- Department of Pediatrics, Faculty of Medicine, Ain-shams University \\ Corresponding author: Khaled Abdelhay, email: km547087@gmail.com
}

\begin{abstract} Care Unit (PICU) of Ain-shams University Hospital. pathogens as causative agent of CLABSI in $9 \%$ of cultures. the common pathogens isolated in CLABSI.

Keywords: CLABSI, Central line, Infection.

\section{INTRODUCTION}

Central line-associated blood stream infections (CLABSIs) are a major source of hospital-acquired infections (HAIs) in PICU and are associated with high morbidity, mortality and also increased economic burden ${ }^{(1)}$. The rate of CLABSI in developing countries ranges from 1.6 to 44.6 cases per 1000 central line (CL) days in $\mathrm{PICU}^{(2)}$. Common pathogens causing CLABSI are staph. Epidermidis (CONS), Methicillin-resistant staph aureus (MRSA), Candida spp, gram-negative bacteria as Klebsiella and Enterobacteriaceae ${ }^{(3)}$. CLABSI has a mortality rate up to $8 \%$, causes longer hospital admission ${ }^{(4)}$ and Increased costs ${ }^{(5)}$.
\end{abstract}

Background: central line-associated blood stream infection (CLABSI) is responsible for almost $60 \%$ of the hospital-acquired infections. The rate of CLABSI in developing countries is higher than that in developed countries. Recently; gram negative bacilli are commonly isolated in CLABSI cases.

Aim of the work: This was a prospective 6 month study to assess the occurrence of CLABSI at Pediatric Intensive

Patients and methods: present study was a prospective observational study conducted on 109 patients with a 152 central venous catheter (CVCs) inserted to them, who were admitted to the Pediatric Intensive Care Unit of Ain Shams University Hospital from July to December 2017. They were observed for development of central lineassociated blood stream infection (CLABSI) and also associated risk factors were studied.

Results: There was 22 CLABSI cases with a CLABSI score 14.1 per 1000 central line days. In this study, the most common organism detected was Klebsiella that was isolated in 14 cases. This study shows multi-drug resistant

Conclusion: CLABSI is a common and serious complication to CVCs in PICU. Klebsiella, candida albicans are

\section{METHODOLOGY}

Study: This study was prospective observational study carried out over six months; from July to December 2017 at pediatric intensive care unit (PICU) of Ain-shams University Hospital.

Patients: This study was carried out on 109 patients (including 152 CVCs) admitted in PICU, All CVCs were inserted under guide of ultrasound.

Inclusion criteria: Patients admitted to PICU and centrally cannulated during their admission as a part of their management.

Exclusion criteria: Patients with infective endocarditis
Full clinical assessment including: Age, sex, duration of PICU admission cause of admission, history of the present illness, duration of CVC, vital signs (temperature, respiratory rate, heart rate and blood pressure), full cardiac, respiratory, abdominal and neurological examination.

Investigations : Complete blood count (CBC), C reactive protein (CRP); initially before insertion of CVC, 48 hrs then $96 \mathrm{hrs}$ following CVC insertion.

Blood culture: Two blood cultures were withdrawn; one from the CVC and the other was peripheral percutaneous blood culture. Cultures are withdrawn at least $48 \mathrm{hrs}$ following CVC insertion especially with appearance of signs of infection.

\section{* Blood Culture technique:}

Manual blood culture system (Conventional) was used: Aerobic blood culture bottles were inoculated with $2 \mathrm{ml}$ blood and usually incubated for 7 days. Each bottle was examined daily for macroscopic evidence of microbial growth (e.g., hemolysis, turbidity of the media, gas production, or formation of discrete colonies). An aliquot of the contents of the aerobic bottle was gram stained and subcultured (Blood and MacConkey Agar) after the first overnight incubation. A terminal subculture was usually done at the end of the incubation period. Conventional manual systems are flexible and require no purchase of expensive instruments, but they are labor intensive ${ }^{(6)}$. 


\section{Detection and recognition of CLABSI}

CLABSI was suspected if the following signs had been noted: Fever $>38$ or hypothermia $<36$, tachycardia, hypotension, poor perfusion, altered mental status or CVC malfunction and confirmed by isolation of the same microorganism from the catheter and concomitant peripheral blood cultures, which are not related to an infection at another site.

\section{Calculation of incidence of CLABSI in our study} was done by CLABSI score

Score $=\{$ number of CLABSIs/total number of CVCdays $\}$ x 1000 .

Results are multiplied by 1,000 ; so that the measure is expressed by the number of CLABSIs) per 1,000 catheter days ${ }^{(7)}$.

\section{Ethical approval}

Verbal consents were obtained from parents of all patients. The study was approved by Aswan University, Faculty of Medicine. The steps of the study, the aim of the study, the potential benefit and hazards, all were discussed with the patient's parents. Confidentiality of all data was ensured.

\section{Statistical Analysis}

Statistical analysis was performed using statistical package for social science "SPSS V21.0, SPSS Inc., Chicago, IL, USA". Continuous data were presented as mean $\pm \mathrm{SD}$ or as median, while categorical variables as percentages. Categorical and continuous variables were analyzed using chi-square test and Mann-whitney test respectively. $\mathrm{P}$ value < 0.05 was considered significant and $\mathrm{P}$ value $<0.01$ was considered highly significant.

\section{RESULTS}

The mean age of the patients in the study sample was $30.34 \pm 24.5$ months. $61 \%$ of patients were males and $39 \%$ were females.

All patients were admitted in PICU; $49.3 \%$ of these patients required mechanical ventilation.
Table (1): Underlying Diagnosis of the studied Cases

\begin{tabular}{|l|c|c|}
\hline \multicolumn{1}{|c|}{ Category } & $\mathbf{N = 1 0 9}$ & \% \\
\hline Respiratory disorders & 31 & $28.4 \%$ \\
\hline Gastrointestinal disorders & 33 & $30.3 \%$ \\
\hline Cardiovascular disorders & 15 & $13.8 \%$ \\
\hline Neuromuscular disorders & 12 & $11 \%$ \\
\hline Renal disorders & 5 & $4.6 \%$ \\
\hline Immunological disorders & 3 & $2.8 \%$ \\
\hline Hematological disorders & 3 & $2.8 \%$ \\
\hline Hepatic disorders & 2 & $1.8 \%$ \\
\hline Metabolic disorders & 2 & $1.8 \%$ \\
\hline Malignancy & 2 & $1.8 \%$ \\
\hline Endocrinal disorders & 1 & $0.9 \%$ \\
\hline
\end{tabular}

Total number of central venous catheter days was 1557 days and the average number of days for which the CVC maintained was $15.4 \pm 13.1$ days. CLABSI was reported in 22 cases, The CLABSI score was 14.1 per 1000 catheter-days, CLABSI occurred in 19 cases when the CVC was inserted into right internal jugular vein and in 3 cases when it was inserted into the left internal jugular vein.

Table (2): showing Comparison between two subgroups of patients (with and without CLABSI) as regards CVC duration, total PICU admission days and initial SOFA score:

\begin{tabular}{|l|c|c|c|c|}
\hline \multirow{2}{*}{ Variable } & & $\begin{array}{c}\text { Without } \\
\text { CLABSI } \\
\text { N=130 }\end{array}$ & $\begin{array}{c}\text { CLABSI } \\
\text { N=22 }\end{array}$ & $\begin{array}{c}\text { P- } \\
\text { value }\end{array}$ \\
\hline \multirow{3}{*}{$\begin{array}{l}\text { CVC } \\
\text { days }\end{array}$} & Mean & $13.99 \pm$ & $22.47 \pm$ & \\
& \pm SD & 9.4 & 11.5 & \multirow{2}{*}{0.021} \\
\cline { 2 - 4 } $\begin{array}{l}\text { Total } \\
\text { PICU }\end{array}$ & Median & $11(3-$ & $\mathbf{2 1}(5-$ & \\
admission & Mean \pm & $16.67 \pm$ & $\mathbf{5 0 . 0 6} \pm$ & \\
\cline { 2 - 4 } days & & 8.4 & 16.2 & \multirow{2}{*}{0.01} \\
\cline { 2 - 4 } & Median & $\mathbf{1 2}(3-$ & $\mathbf{2 4}(5-$ & \\
\hline
\end{tabular}

PICU: pediatric intensive care unit, CVC: central venous catheter, CLABSI: central line associated bloodstream infection. 


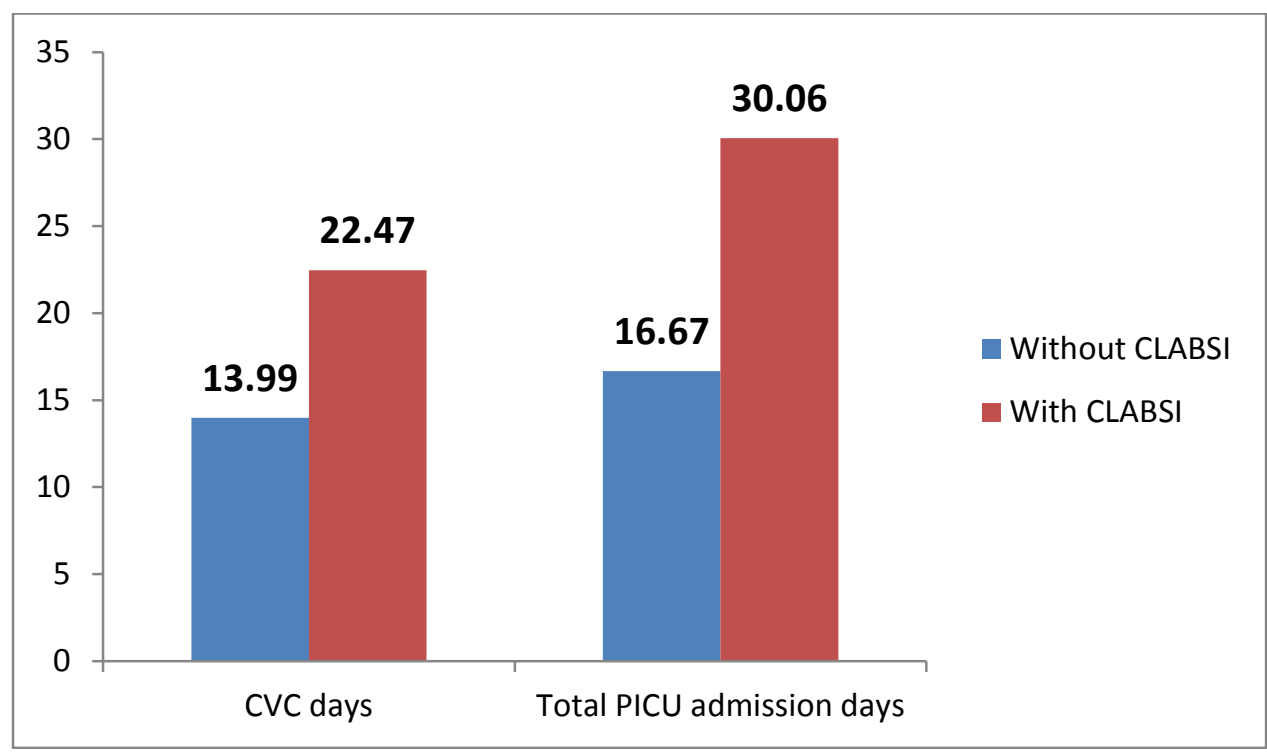

Fig. (1) : Bar chart showing Comparison between two subgroups of patients (with and without CLABSI ) as regards CVC duration and total PICU admission days.

Table (3): Comparison between two subgroups of patients (with and without CLABSI) as regards CVC site:

\begin{tabular}{|l|c|c|c|}
\multicolumn{1}{|c|}{ Site } & $\begin{array}{c}\text { Non-CLABSI } \\
\text { N= 130 }\end{array}$ & $\begin{array}{c}\text { CLABSI } \\
\mathbf{N = 2 2}\end{array}$ & \multirow{2}{*}{ P-value } \\
\hline Rt IJV & $88(67.6 \%)$ & $19(86.4 \%)$ & \\
\cline { 1 - 3 } Lt IJV & $33(25.4 \%)$ & $3(13.6 \%)$ & \multirow{2}{*}{$>0.05$} \\
\cline { 1 - 3 } Rt Femoral & $4(3.1 \%)$ & $0(0 \%)$ & \\
\cline { 1 - 3 } Lt femoral & $4(3.1 \%)$ & $0(0 \%)$ & \\
\cline { 1 - 3 } Rt Subclavian & $1(0.8 \%)$ & $0(0 \%)$ & \\
\hline
\end{tabular}

\section{CLABSI: Central Line -Associated Blood Stream Infections IJV: internal jugular vein}

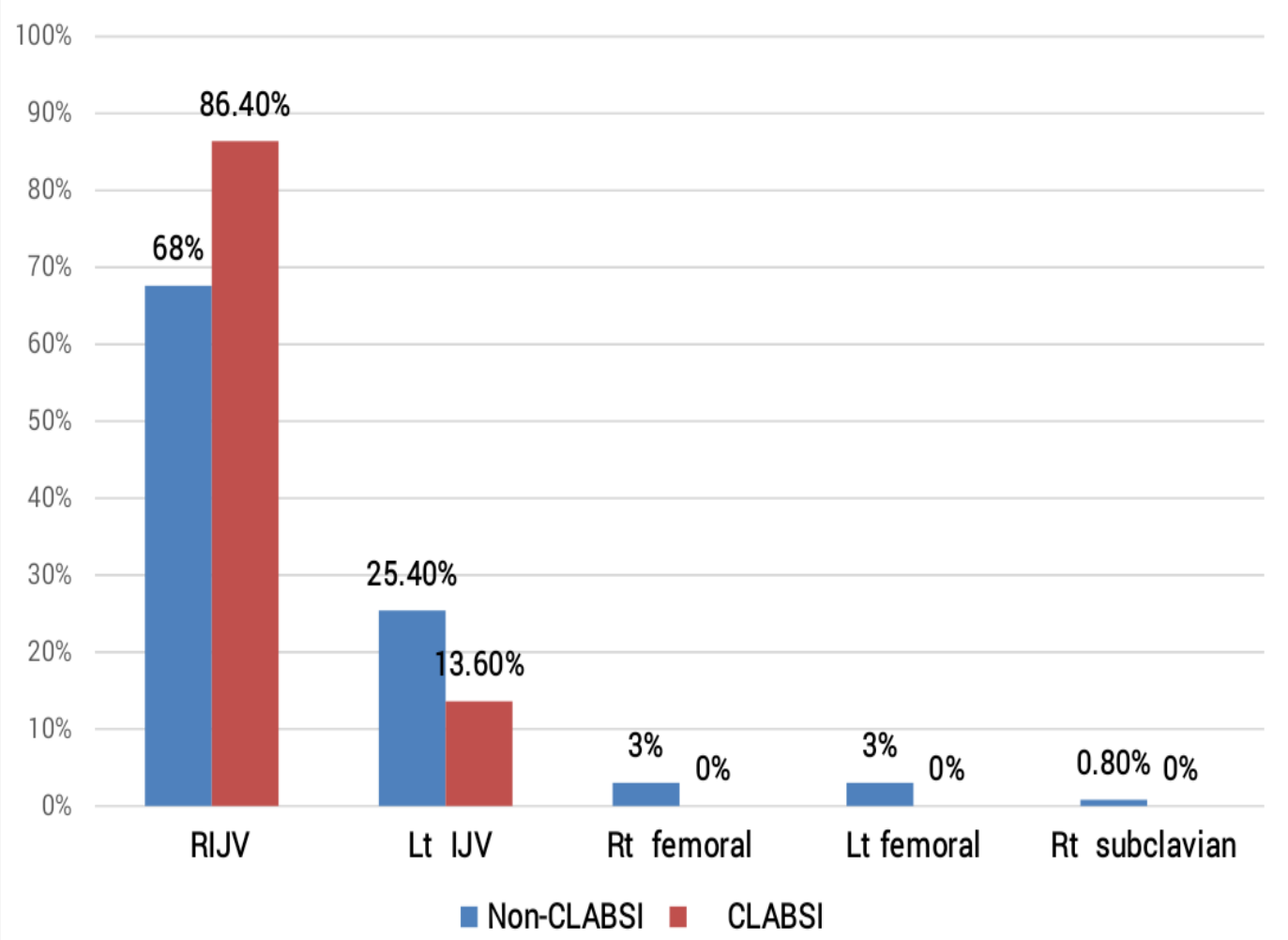

Fig. (2): Bar chart comparing between two subgroups of patients (with and without CLABSI) as regards CVC site. 
Organisms reported are shown in table 4. .

Table (4): Frequency of organisms detected in CLABSI cases:

\begin{tabular}{|l|c|l|}
\hline Causative organism & $\begin{array}{c}\text { Frequency } \\
\mathbf{N = 2 2}\end{array}$ & \% \\
\hline Klebsiella & 14 & $63.6 \%$ \\
\hline Candida albicans & 3 & $13.6 \%$ \\
\hline Proteus & 2 & $9.1 \%$ \\
\hline MRSA & 2 & $9.1 \%$ \\
\hline E. coli & 1 & $4.6 \%$ \\
\hline Total CLABSI cases & 22 & $100 \%$ \\
\hline
\end{tabular}

MRSA: Methicillin-resistant staph. Aureus; CLABSI: Central Line-Associated Bloodstream Infection.

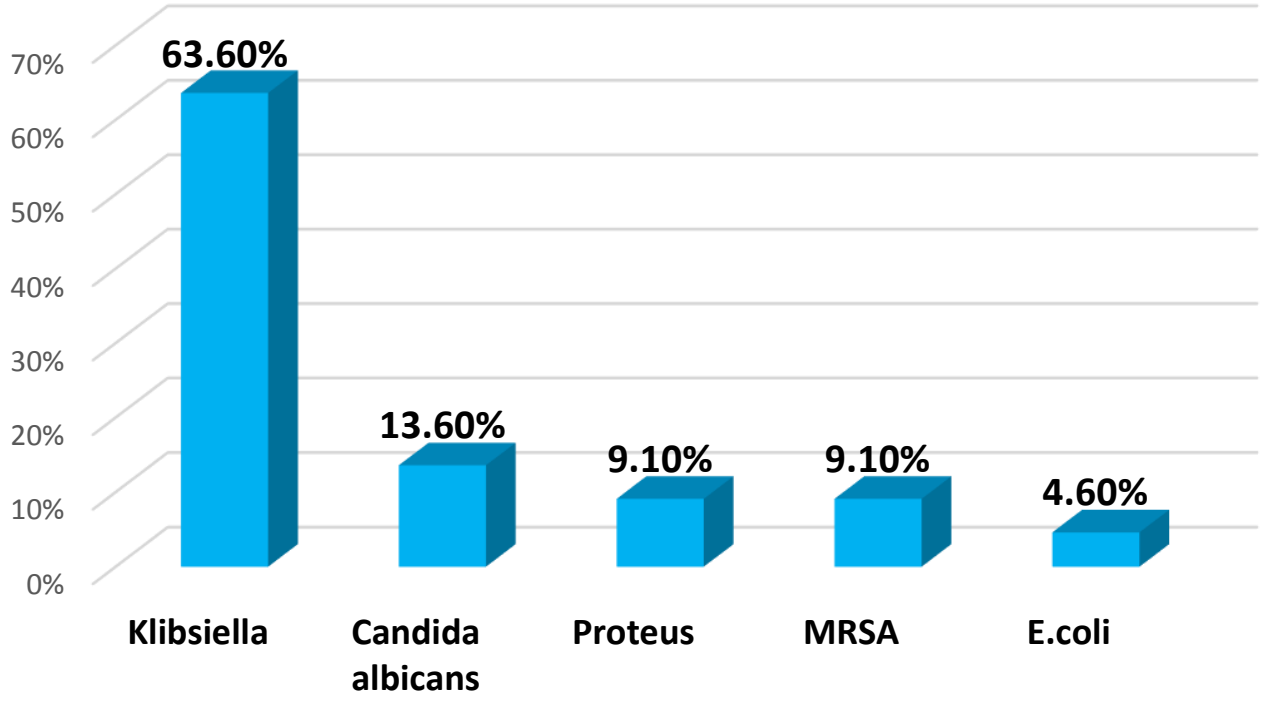

Causative organism

Fig. (3): Bar chart shows frequency of organisms detected in CLABSI cases;

Results of the sensitivity are shown in table 5 .

Table (5): showing pattern of sensitivity to Klebsiella:

\begin{tabular}{|l|c|}
\hline \multicolumn{1}{|c|}{ Antibiotic } & \% \\
\hline Ampicillin/sulbactam & $12.7 \%$ \\
\hline Amoxicillin/clavulanate & $9 \%$ \\
\hline Cefotaxime & $9 \%$ \\
\hline Ceftriaxone & $12.7 \%$ \\
\hline Cefepime & $9 \%$ \\
\hline Amikacin & $29 \%$ \\
\hline Gentamycin & $12.7 \%$ \\
\hline Meropenem & $7.3 \%$ \\
\hline Imipenem & $7.3 \%$ \\
\hline Ciprofloxacin & $5.5 \%$ \\
\hline Levofloxacin & $7.3 \%$ \\
\hline Teicoplanin & $7.3 \%$ \\
\hline Cefoperazone/sulbactam & $41.8 \%$ \\
\hline MDRO & $9 \%$ \\
\hline
\end{tabular}


ejhm.journals.ekb.eg

Table (6): Showing the relation between CLABSI with mortality:

\begin{tabular}{|c|c|c|}
\hline Category & CLABSI & \% \\
\hline Total number & 22 & $100 \%$ \\
\hline Survivors & 14 & $63.6 \%$ \\
\hline Non-survivors & 8 & $36.4 \%$ \\
\hline
\end{tabular}

\section{DISCUSSION}

In our study the CLABSI score was 14.1 per 1000 catheter-days while a study in India reported a 7.9 per 1000 catheter-days ${ }^{(8)}$. Other studies from hospitals in India reported a CLABSI rate of 2.8 and $18.5 \%$ per 1000 catheter days respectively ${ }^{(9,10)}$. Centers for Disease Control and Prevention (CDC) in USA have detected 30,100 CLABSI cases in Intensive Care Units (ICU) each year ${ }^{(11) .}$

As regard The Site of CVC; no statistically significant relation had been found between CVC site and CLABSI development. This is consistent with a study by Timsit et al. ${ }^{12}$ that found no difference in CLABSI or CVC colonization between internal jugular vein and femoral vein $\mathrm{CVCs}^{(12)}$. These results are different from other studies that do not prefer to use femoral site for CVC insertion because of their higher rates of infectious complications compared to the internal jugular and subclavian sites ${ }^{(13,14)}$.

As regard CVC duration; our study showed that duration of CVC was a significant factor in developing CLABSI. Same results was found by Wylie et al. ${ }^{15}$ who found that increase CVC duration lead to increase incidence of CLABSI ${ }^{(15)}$. Also, total PICU admission days had a significant relation with CLABSI in our study with.

Organisms reported in CLABSI was Klebsiella 14 (63.6\%), candida albicans $3(13.6 \%)$, proteus in 2 cases $(9.1 \%)$, MRSA in 2 cases $(9.1 \%)$, E.coli in only one case $(4.6 \%)$. Inconsistent with other studies in which the most common organisms cultured in CLABSI cases were coagulase-negative staphylococci (CONS) ${ }^{(13)}$ and a study by Newman showed that most common causative pathogens for CLABSI in the US are CONS, S. aureus, enterococci, and Candida $\operatorname{spp}^{(16)}$, but similar to other studies that found that the gram negative bacilli represented 96.5\% of bacterial isolates causing healthcareassociated infections ${ }^{(17,18)}$.

\section{CONCLUSION}

- The CLABSI score was 14.1 per 1000 catheter-days; similar to incidence of CLABSI in developing countries but largely higher than developed countries as USA.

- CLABSI was associated with high risk of mortality.

- Klebsiella was the most common organism reported in CLABSI cases.

\section{REFERENCES}

1. Nowak J, Brilli R, Lake M et al. (2010): Reducing catheter-associated bloodstream infections in the pediatric intensive care unit: business case for quality improvement. Pediatr Crit Care, 11:579-87.

2. Rosenthal VD (2009): Central line-associated bloodstream infections in limited-resource countries: a review of the literature. Clin. Infect Dis., 49:1899907.

3. Tille P and Scott S (2013): Diagnostic Microbiology, 13th Ed. New York: Elsevier.

4. Chesshyre E, Goff Z, Bowen et al. (2015): The prevention, diagnosis and management of central venous line infections in children. J Infect., 71:S5975.

5. Zimlichman E, Henderson D, Tamir O et al. (2013): Health care-associated infections: a metaanalysis of costs and financial impact on the US health care system. JAMA internal medicine, 173(22):203946.

6. shore $A$ and sandoe $\mathbf{J}$ (2008) : blood cultures . student BMJ. , 16 : 324-325.

7. Centers for Disease Control and Prevention.(2015): Bloodstream Infection Event (Central Line-Associated Bloodstream Infection and Non-central line associated Bloodstream Infection). Available from: http:// www. cdc. gov/nhsn/PDFs/pscManual/4PSC_CLABS current.

8. Mehta A, Rosenthal VD, Mehta Y et al. (2007): Device-associated nosocomial infection rates in intensive care units of seven Indian cities. Findings of the International Nosocomial Infection Control Consortium (INICC). J Hosp Infect., 67:168-74.

9. Patil H, Patil V, Ramteerthkar M et al. (2011): Central venous catheter-related bloodstream infections in the intensive care unit. Indian J Crit Care Med. , 15:213-2.

10. Kaur R, Mathai A and Abraham J (2012): Mechanical and infectious complications of central venous catheterizations in a tertiary-level intensive care unit in northern India. Indian J. Anaesth. , 56:376-81.

11. Centers for disease control and prevention/NHSN. (2011): Guidelines for the prevention of intravascular catheter related infections. https://www.cdc.gov > hai > pdfs > bsi-guidelines-2011

12. Timsit JF, Rupp M, Bouza E et al. (2018): A state of the art review on optimal practices to prevent, recognize, and manage complications associated with intravascular devices in the critically ill. Intensive Care Med., 44(6):742-759.

13. O'grady NP, Alexander M, Burns LA et al. (2011): Guidelines for the prevention of intravascular 
ejhm.journals.ekb.eg

catheter-related infections. Clinical infectious diseases, 52(9):e162-93.

14. Parienti JJ, Mongardon N, Mégarbane B et al. (2015): Intravascular complications of central venous catheterization by insertion site. N. Engl. J. Med., 373(13):1220-9.

15. Wylie MC, Graham DA, Potter-Bynoe G et al. (2010): Risk factors for central line-associated bloodstream infection in pediatric intensive care units. Infect Control Hosp Epidemiol., 31(10):1049-56.
16. Newman C (2006): Catheter-related bloodstream infections in the pediatric intensive care unit. Semin Pediatr Infect Dis., 17:20-4.

17. Lakshmi KS, Jayashree M, Singhi S et al. (2006): Study of nosocomial primary bloodstream infections in a pediatric intensive care unit. J Trop Pediatr., 53:87-92.

18. Gupta A, Kapil A, Lodha $R$ et al. (2011): Burden of healthcare-associated infections in a paediatric intensive care unit of a developing country: A single centre experience using active surveillance. J Hosp Infect., 78:323-6. 